\title{
Automated Mottling Assessment of Colored Printed Areas
}

\author{
Albert Sadovnikov, Lasse Lensu, and Heikki Kälviäinen \\ Machine Vision and Pattern Recognition Group \\ Laboratory of Information Processing \\ Department of Information Technology \\ Lappeenranta University of Technology \\ P.O.Box 20, 53851 Lappeenranta, Finland \\ Firstname.Lastname@lut.fi \\ http://www.it.lut.fi/ip/research/mvpr/
}

\begin{abstract}
Mottling is one of the most significant defects in modern offset printing using coated papers. Mottling can be defined as undesired unevenness in perceived print density. Previous research in the field considered only gray scale prints. In our work, we extend current methodology to color prints. Our goal was to study the characteristics of the human visual system, perform psychometric experiments and develop methods which can be used at industrial level applications. We developed a method for color prints and extensively tested it with a number of experts and laymen. Suggested approach based on pattern-color perception separability proved to correlate with the human evaluation well.
\end{abstract}

\section{Introduction}

Paper printability and quality of prints are important attributes of modern printing applications. These issues are of equal significance for all parties involved in printed media production, from paper mills and print houses to end-consumers. High print quality is a basic requirement in printed products containing images. There are several undesired effects in prints because of non-ideal interactions of paper and ink in high-speed printing processes. One of these effects is mottling which is related to density and gloss of print. It is the uneven appearance of solid printed areas, and it depends on the printing ink, paper type, and printing process. Depending on the phenomenon causing this unevenness, there exist three types of mottling: back-trap mottle (uneven ink absorption in the paper), water-interface mottle (insufficient and uneven water absorption of the paper causing uneven ink absorption), and ink-trap mottle (wet or dry; incorrect trapping of the ink because of tack. In the task of automated mottling measurement the main cause of mottling is often overlooked, namely the human perception. Truly, if a person could not perceive printed area unevenness then the attention to mottling would be a lot less. 
Several methods to evaluate mottling by an automatic machine vision system have been proposed. The ISO 13660 standard includes a method for monochrome images. It is based on calculating the standard deviation of small tiles within sufficiently large area [1. In the standard, the size of the tiles is set to a fixed value, which is a known limitation [2. The first improvement to the standard method was to use tiles of variable sizes [3. Other methods relying on clustering, statistics, and wavelets have also been proposed [4.56]. Other approaches to evaluate gray scale mottling have their basis in frequency-domain filtering [7] and frequency analysis [8, which were thoroughly studied in [9]. All of the beforementioned methods are designed for binary or gray scale images. If color prints were assessed, the correlation of the methods to human assessments would be severely limited. Also the grounds for the methods do not arise from any models for the phenomena causing mottling, nor vision science.

Mottling can be physically defined, but it becomes problematic when a print is perceived. If a person looking at a solid print perceives unevenness, mottling is a problem. Thus, the properties and limits of the human visual system must be taken into account when proper methods to assess mottling are designed. This is especially very important in the assessment of color images. When perception of image noise is of concern, visual sensitivity to contrast and spatial frequencies of the human visual system (HVS) is independent of luminance within common luminance levels [10]. However, contrast sensitivity depends on spatial frequency 11, thus, mottles of different sizes are perceived differently. The peak sensitivity of the HVS is approximately at 3 cycles/degree, and the maximum detected frequency is from 40 cycles/degree (sinusoidal gratings) [12 to over 100 cycles/degree (single cycle) 13 .

The purpose of this work was to design the artificial method for a human assessment of color mottling samples. In our study, we sought proper background for the methodological selections based on vision science. We implemented the method based on gray scale approach, and modified for color prints, as needed to accommodate appropriate knowledge concerning the HVS.

\section{Background}

Method presented in the paper is based on the current approaches to gray scale mottling evaluation and pattern-color separability hypothesis. It is worth to give a short introduction to those.

\subsection{Gray Scale Mottling Evaluation}

The human perception of mottling relies on the spatial frequency theory, which is based on an atomistic assumption: the representation of any image, no matter how complex, is an assemblage of many primitive spatial "atoms". The primitives of spatial frequency theory are spatially extended patterns called sinusoidal 
gratings: two dimensional patters whose luminance varies according to a sine wave over one spatial dimension and is constant over the perpendicular dimension [14.

The idea for this method comes from the bandpass method [7 and later extended in 9]. The value of the perceived mottling can be computed using the following formula:

$$
M=\frac{1}{R} \int_{u \in U, \phi \in \Phi}|F(u, \phi)| C(u) d u d \phi
$$

where $F(u, \phi)$ image in frequency space, $C(u)$ contrast sensitivity function (CSF), $R$ is a mean image reflectance. The implementation of the method uses Mannos CSF defined as [15:

$$
C(u)=2.6(0.0192+0.114 u) e^{\left(-(0.114 u)^{1.1}\right)}
$$

Mannos CSF formulation is in cycles per degree (cpd) units and has a maximum at $8 \mathrm{cpd}$ (see Fig. 1).

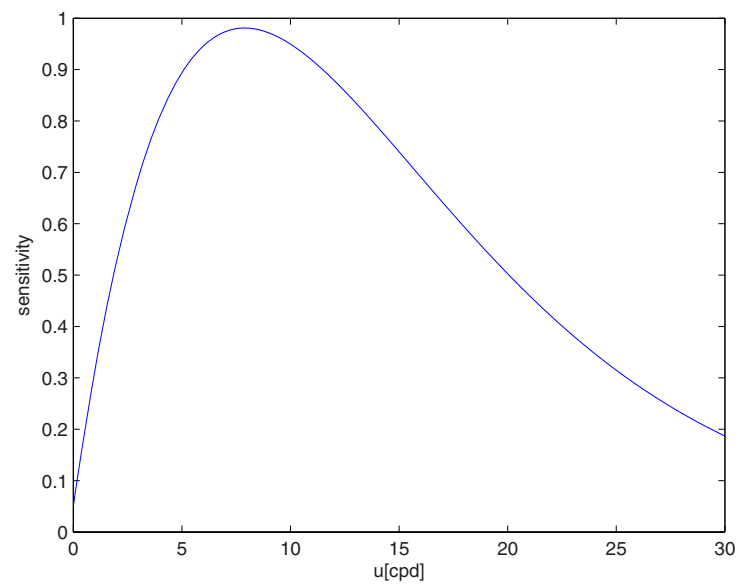

Fig. 1. Mannos contrast sensitivity function model

The method can be defined as a sum of the image energy filtered with CSF in the spectrum that falls within the visual area, which is $U=[0.04,30] \mathrm{cpd}$ in the current implementation.

The effect of orientation $(\phi)$ sensitivity of the has also been studied HVS [12]. It is known that human sensitivity is lowest around 45 and 135 and highest at vertical and horizonal directions. However, experiments showed low significance of introducing the orientational dependent filtering. This can also be understood based on the stochastic nature of mottling. 


\subsection{Pattern-Color Separability}

A mechanism of color mottling stimuli perception is pattern-color separable when [16]:

- Its relative pattern sensitivity is invariant as the test stimulus' spectral composition is changed.

- Its relative wavelength selectivity is invariant when stimulus' spatial pattern is changed.

The absolute level of the pattern and color sensitivity can vary, but the relative pattern and color sensitivities must not. From this definition it is evident that pattern-color separability is required before it is possible to say that a mechanism has a unique pattern or wavelength sensitivity function. The pattern-color separable model provides a framework for thinking about how different visual pathways might contribute to visual sensitivity [16.

If the previous ideas are right, then color mottling perception depends separately on the spatial characteristics of an image (gray scale mottling) and color characteristics (color range). The following section will describe how it is possible to decompose an image in the pattern and color sense.

\section{Pattern-Color Separable Method}

Let us consider a gray scale image $f(x, y)$ with a given mottling index $M$. The question arises, how the mottling perception will change if the image will be colored with the following coloring procedure: red component $f_{r}(x, y)=$ $c_{r} f(x, y)+m_{r}$, green component $f_{g}(x, y)=c_{g} f(x, y)+m_{g}$ and blue component $f_{b}(x, y)=c_{b} f(x, y)+m_{b}$. It is clear that perceived mottling value will depend on the pattern $f(x, y)$ and on the visual color differences along the line $\left(m_{r}, m_{g}, m_{b}\right)+k\left(c_{r}, c_{g}, c_{b}\right)$. This also holds for the gray scale perception model (see Eq. 11), where mottling value depends on the mean reflectance $R$ (like $\left.\left(m_{r}, m_{g}, m_{b}\right)\right)$, but since the color orientation in gray level images is constant $(1,1,1)$, it is not used.

Basically, the $1 / R$ factor in Eq. 1 reflects simplified correspondence between lightness and luminance, making color difference along the line $(1,1,1)$ locally constant. This fact leads to an idea to use a perceptually uniform color system for computing mottling index, where the role of the mean sample color and the color orientation will be reduced to a minimum. For the method design 1976 CIE $L^{*} a^{*} b^{*}$ color space was used.

Color mottling evaluation can be summarized in Algorithm 1

\section{Algorithm 1. Pattern-color separable method}

1: Transform an image from the input color space to 1976 CIE $L^{*} a^{*} b^{*}$.

2: Compute at each plane $\left(L^{*}, a^{*}, b^{*}\right)$ mottling value with Eq, 1 , omitting $1 / R$ factor.

3: Compute the final mottling index as $M=\sqrt{M_{L}^{2}+M_{a}^{2}+M_{b}^{2}}$. 


\section{Human Evaluation}

\subsection{Pairwise Comparison Test}

The basic method of paired comparisons 17 consists of sequentially presenting pairs of samples to an observer and asking the observer which one of the pair has the greatest amount of mottling.

For this test we used a set of 62 black at $70 \%$ (K70) samples, with previously known and tested mottling values [18, covering a wide range of mottling. It is obviously impossible to evaluate a perception model for mottling using only K70 (gray scale samples). To extend the sample set, the following coloring procedure was used:

$$
\left(f_{r}(x, y), f_{g}(x, y), f_{b}(x, y)\right)=\left(c_{r} f(x, y)+m_{r}, c_{g} f(x, y)+m_{g}, c_{b} f(x, y)+m_{b}\right)
$$

where $f(x, y)$ is an input K70 sample, $f_{r}, f_{g}, f_{b}$ - new image color planes, $m_{r}, m_{g}, m_{b}$ - new image mean color value, and vector $\left(c_{r}, c_{g}, c_{b}\right)$ describes image color range orientation in red-green-blue (RGB) color space and also includes scaling effect, i.e., vector $\left(c_{r}, c_{g}, c_{b}\right)$ is not normalized.

Since it is not feasible to cover all the visible color space, the following areas of interest were used: cyan at 70\% (C70), magenta at 70\% (M70), yellow at $70 \%$ (Y70), K70. These inks are usually used as printing primaries, and therefore are of more interest.

Pairwise comparison test can be summarized in the Algorithm 2

\section{Algorithm 2. Pairwise comparison test}

1: Select randomly two samples from Kr0 set.

2: Select mean color $m_{r}, m_{g}, m_{b}$, i.e. C70, Mro, Yro or Kro.

3: Randomly generate two color orientation vectors of the form $\left(c_{r}, c_{g}, c_{b}\right)$.

4: Color each sample with previously defined mean color and orientations.

5: Present a pair for evaluation.

When presented a pair (see the test layout in Fig. 2) an observer is asked to give a mark for the difference between the samples, ranged in $-3,-2,-1,0,1,2$, 3. The test is organized in the way which allows to avoid color adaptation, i.e. saturated samples (C70, M70 and Y70) are alternated with unsaturated (K70).

The observers were divided into two groups: experts and laymen. Experts were individuals with vast mottling evaluation experience(10 persons), and others were defined as laymen(15 persons). Each pair evaluation was limited by a certain time (15 seconds), to avoid such artifacts as pattern/color adaptation and guessing.

\subsection{Reference Set}

Current mottling evaluation practise is based on experts opinion. Given a mottling sample, an expert is asked to put it into a certain predefined category, 


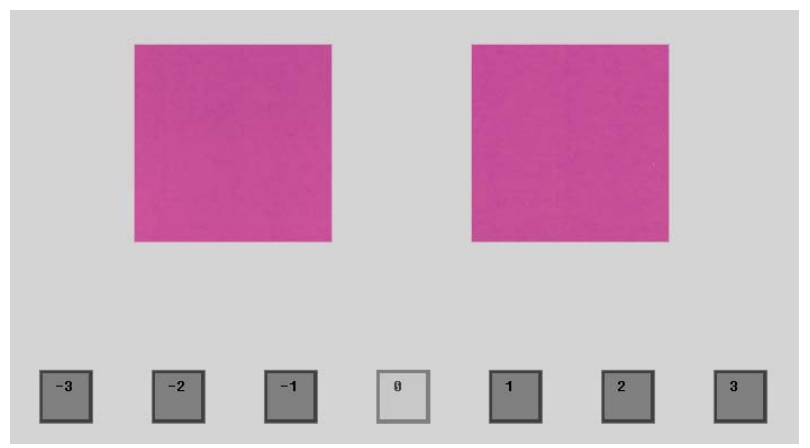

Fig. 2. Pairwise comparison test layout

based on the perceived mottling characteristics. The number of categories vary from a party involved (paper manufacturers, printing houses).

Grading a mottling sample requires a reference set, i.e., a set of samples with different levels of mottling. This set is constructed basing on several experts' experience and makes the evaluation procedure straightforward: an evaluated sample is placed among the reference samples and given a category of the closest reference sample.

For the performance testing of our method we used 10 reference sets, 5 for cyan at 50\% (C50) and 5 for black at 50\% (K50). C50 reference set consisted of 7 different classes of mottling, ranged from 0 to 6 , and K50 sample set ranged from 1 to 6, making it 35 C50 samples and 30 K50 samples. Smaller values correspond to less uneven images.

Given reference samples were used in correlation computation, in method performance evaluation, and in separability checks.

\section{Parameter Estimation}

The architecture of the method presented in Section 3 is relatively simple. However it contains a number of parameters that need to be estimated carefully for better performance.

For a feasible and relatively simple analysis we will use so called correlation images [19. Consider a pairwise test, described in Section 4.1. Denote $i^{\text {th }}$ pair of images as $f_{i}(x, y)$ and $g_{i}(x, y)$. Corresponding pair of Fourier magnitudes will be $\left|F_{i}(u, v)\right|$ and $\left|G_{i}(u, v)\right|$. Difference in mottling values for this, marked by an observer will be $H_{i}$. If our model is consistent, then (simplified case)

$$
\begin{gathered}
\int_{u, v}\left|F_{i}(u, v)\right| C(u, v) d u d v-\int_{u, v}\left|G_{i}(u, v)\right| C(u, v) d u d v \sim H_{i} \\
\int_{u, v}\left(\left|F_{i}(u, v)\right|-\left|G_{i}(u, v)\right|\right) C(u, v) d u d v \sim H_{i}
\end{gathered}
$$

and in the discrete case:

$$
\sum_{u, v}\left(\left|F_{i}(u, v)\right|-\left|G_{i}(u, v)\right|\right) C(u, v) \sim H_{i}
$$


If we make an assumption about the cross frequency independence, i.e., certain frequency component of an image can not be estimated with other frequency components, then the amount of information introduced by each frequency point to the perceived mottling should correspond to the contrast sensitivity at this point. We measure this information by using correlation image $R_{H}(u, v)$ for the perceived difference $H$ ( $N$ stands for the number of pairs):

$$
R_{H}(u, v)=\operatorname{corr}\left(\left\{\left|F_{i}(u, v)\right|-\left|G_{i}(u, v)\right|\right\}_{1}^{N},\left\{H_{i}\right\}_{1}^{N}\right)
$$

In our case, since the amount of information introduced by each of the planes $L^{*}, a^{*}$ and $b^{*}$ to the perception model is assumed to be equal (color space uniformity), we assume the following:

$$
\begin{aligned}
R_{H}\left(u_{L}, v_{L}\right) & \sim C\left(u_{L}, v_{L}\right) \\
R_{H}\left(u_{a}, v_{a}\right) & \sim C\left(u_{a}, v_{a}\right) \\
R_{H}\left(u_{b}, v_{b}\right) & \sim C\left(u_{b}, v_{b}\right)
\end{aligned}
$$

which means that correlation images of each color plane should model the correlation function to some extent. In Fig. 3 it can be noticed that correlation planes yield similar information in terms of correlation of certain frequency bands with perceived mottling. In can be also seen that $L^{*}$ correlation plane has more

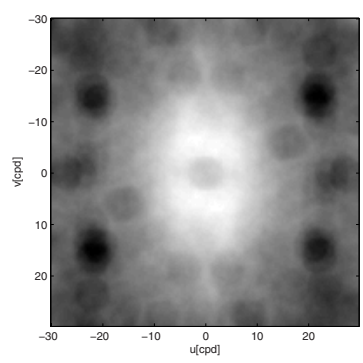

(a)

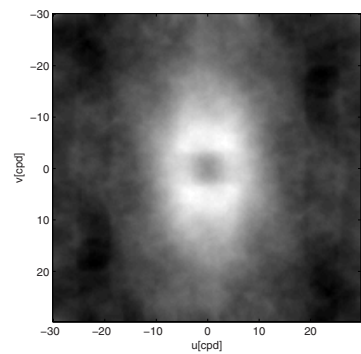

(b)

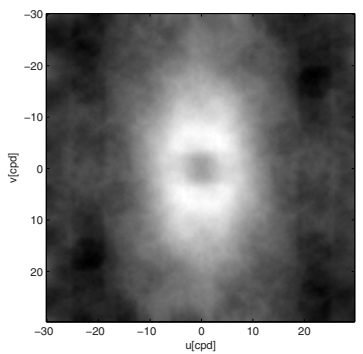

(c)

Fig. 3. Correlation planes: (a) $L^{*}$ plane; (b) $a^{*}$ plane; (c) $b^{*}$ plane 


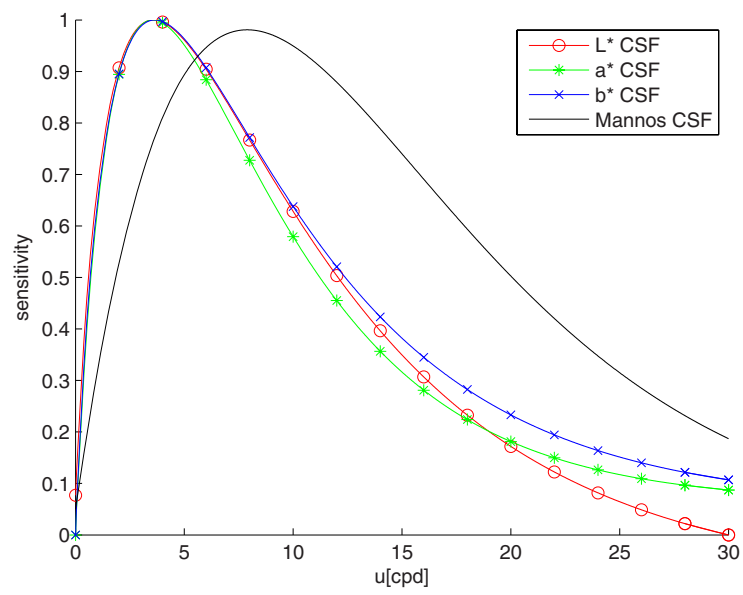

Fig. 4. Contrast sensitivity function models

information and this is probably due to linear nature of random stimuli generation. However the relative energy distribution is similar to those in $a^{*}$ and $b^{*}$ planes.

Consequently, if we modify Mannos CSF to follow the correlation planes, we can derive for each plane separate CSF by fitting the curve parameters. The derived functions are presented in Fig 4 It can be concluded, that the patterncolor separable model works well in this case of stimuli, because the derived sensitivity curves are similar.

\section{Results and Conclusion}

We have computed correlations of the methods' performances in the case of pairwise test and reference set. The numbers are comprised in the Table 1. It is clear that pattern-color separable method outperforms the gray scale one because of the simple reason: it includes information from the $a^{*}$ and $b^{*}$ planes in a way relevant to HVS.

Extremely good performance on the reference sets can be explained by a big relative mottling scale distance between samples, and a $100 \%$ agreement between experts on a samples' classification. However, from Fig. 5 it can be seen that in case of gray scale method, mottling classes 3 and 4 are not quite clearly separated.

The overall performance of the pattern-color separable method can be described as extremely promising, though there is still a room for improvements. Used CSF formulations can be replaced by the most recently developed ones [10]. Also, the perceptual uniformity of the $1976 C I E L^{*} a^{*} b^{*}$ color space is questionable [20]. 
Table 1. Mottling assessment correlations

\begin{tabular}{|l|c|c|c|c|}
\hline Methods & Laymen (1100 pairs) & Experts (300 pairs) & Overall (1400 pairs) & Reference \\
\hline Gray scale & 0.73 & 0.77 & 0.75 & 0.98 \\
Pattern-Color & 0.82 & 0.87 & 0.85 & 0.99 \\
\hline
\end{tabular}

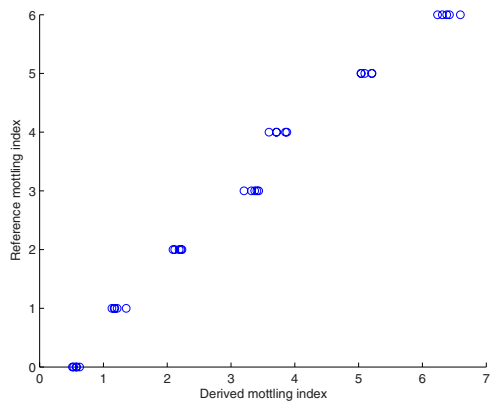

(a)

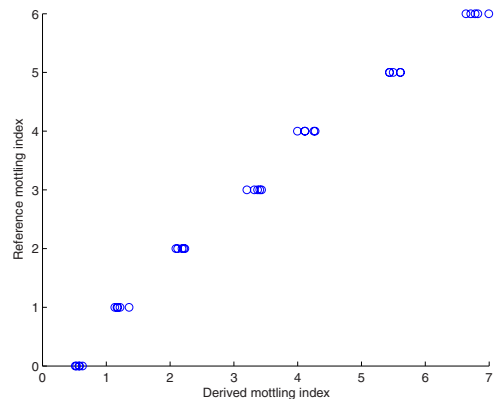

(b)

Fig. 5. Reference mottling indexes versus derived with: (a) Gray scale method; (b) Pattern-Color separable method

\section{Acknowledgments}

This work was done as a part of Papvision project funded by European Union, National Technology Agency of Finland (TEKES Projects No. 70049/03, 70056/04, 40483/05), Academy of Finland (Project No. 204708), and Research Foundation of Lappeenranta University of Technology.

\section{References}

1. ISO/IEC: 13660:2001(e) standard. information technology - office equipment - measurement of image quality attributes for hardcopy output - binary monochrome text and graphic images. ISO/IEC (2001)

2. Briggs, J., Forrest, D., Klein, A., Tse, M.K.: Living with ISO-13660: Pleasures and perils. In: IS\&Ts NIP 15: 1999 International Conference on Digital Printing Technologies, IS\&T, Springfield VA, pp. 421-425 (1999)

3. Wolin, D.: Enhanced mottle measurement. In: PICS 2002: IS\&T's PICS conference, IS\&T pp. 148-151 (2002)

4. Armel, D., Wise, J.: An analytic method for quantifying mottle - part 1. Flexo pp. 70-79 December 1998 (1998)

5. Armel, D., Wise, J.: An analytic method for quantifying mottle - part 2. Flexo, January 1999, pp. 38-43 (1999)

6. Streckel, B., Steuernagel, B., Falkenhagen, E., Jung, E.: Objective print quality measurements using a scanner and a digital camera. In: DPP 2003: IS\&T International Conference on Digital Production Printing and Industrial Applications. pp. 145-147 (2003) 
7. Johansson, P.Å.: Optical Homogeniety of Prints. PhD thesis, Kunglika Tekniska Högskolan, Stockholm (1999)

8. Rosenberger, R.R.: Stochastic frequency distribution analysis as applied to ink jet print mottle measurement. In: IS\&Ts NIP 17: 2001 International Conference on Digital Printing Technologies, IS\&T, Springfield VA, pp. 808-812 (2001)

9. Sadovnikov, A., Lensu, L., Kamarainen, J., Kalviainen, H.: Quantifed and perceived unevenness of solid printed areas. In: Xth Ibero-American Congress on Pattern Recognition, Havana, Cuba, pp. 710-719 (2005)

10. Barten, P.: Contrast Sensitivity of the Human Eye and its Effects on Image Quality. SPIE (1999)

11. Schade, O.H.: Optical and photoelectric analog of the eye. Journal of the Optical Society of America 46, 721-739 (1956)

12. Kang, H.R.: Digital Color Halftoning. SPIE IEEE Press, New York (1999)

13. Campbell, F.W., Carpenter, R.H.S., Levinson, J.Z.: Visibility of aperiodic patterns compared with that of sinusoidal gratings. Journal of Physiology pp. 204 283-298

14. Palmer, S.: Vision Science: Photons to Phenomenology, 1st edn. MIT Press, Cambridge (1999)

15. Mannos, J., Sakrison, D.: The effects of a visual fidelity criterion on the encoding of images. IEEE Transactions on Information Theory 20(4), 525-536 (1974)

16. Poirson, A., Wandell, B.: Pattern-color separable pathways predict sensitivity to simple colored patterns. Vision Research 36, 515-526 (1996)

17. Engeldrum, P.G.: Psychometric Scaling: A Toolkit For Imaging Systems Development. 1st edn. Imcotec Press (2000)

18. Sadovnikov, A., Salmela, P., Lensu, L., Kamarainen, J., Kalviainen, H.: Mottling assessment of solid printed areas and its correlation to perceived uniformity. In: 14th Scandinavian Conference of Image Processing, Joensuu, Finland, pp. 411-418 (2005)

19. Sadovnikov, A., Lensu, L., Kämäräinen, J., Kälviäinen, H.: Model based generation of random stimuli and parameter analysis for print unevenness evaluation. In: Proceedings of the 29th European Conference on Visual Perception, St. Petersburg, Russia, pp. 49-50 (2006)

20. Johnson, G., Fairchild, M.A.: A top down description of S-CIELAB and CIEDE2000. Color Research and Application 28(6), 425-435 (2003) 